value of these well-produced books lies in the good coverage of each topic and in the extensive bibliographies at the end of each chapter. All are well worth reading, for, even if the reader has not had occasion to use the techniques deseribed, a potential application may well become apparent.

K. W. BAGNALL

\section{IS YOUR JOURNAL REALLY NECESSARY?}

Survey of Progress in Chemistry

Vol. 1. Edited by Arthur F. Scott. Pp. xii +340. (New York: Academic Press, Inc.; London: Academic Press, Inc. (London), Ltd., 1963.) $64 s$.

$\mathrm{N}$ his preface the editor of this book states that his aim 1 is "to improve the transmission of new material to the college chemistry teacher". Borrowing a simile from $J$. D. Bernal, he views the traditional dissemination of chemical information through scientific journals as being on a par with the most primitive level of wind pollination in certain plants. He hopes that the Survey will provide a more advanced mechanism corresponding to the selective stage of insect pollination where with far less pollen more gets to the right flowers. The flowers he has in mind are the slightly faded or highly specialized blooms of those who completed their graduate studies about ten vears ago. The articles in the Survey are not intended to be the usual type of review article but are meant to let chemists know what is happening outside their own fields of specialized activity.

Volume l contains seven articles. The first one by Riley Schaeffer is entitled "New Research Tools of Chemists" and deals with nuclear magnetic resonance, electron spin resonance, mass spectrometry. X-ray, neutron and electron diffraction, spectrosenpy and optical rotatory dispersion, all in 33 pages. The principles, principal uses and limitations of each method are clearly and concisely described; but several times the reader has to be content with a promise that one or other of the methods will have a whole article to itself in a future volume of the series. All in all, this was a satisfying hors-d'cuvres, tasty and varied but leaving one asking for more.

The second chapter on "High-Temperature Reactions" is by Alan W. Searcy. It describes the application of thermodynamics to high-temperature inorganic reactions. The important part that entropy plays is quite rightly emphasized. The way in which heats of formation for a series of compounds, for example, the oxides, vary across the periodic table is described and discussed. By combining these $\Delta H$ and $\Delta S$ terms patterns of behaviour for different metals with one or two non-metals can be discerned and explained. This is an excellent article, which brings fresh insight and order to the field of inorganic chemistry by applying simple thermodynamic concepts, which the college teacher should still remember from those semesters ten years ago.

Another excellent article is "Structure and Valence" by R. E. Rundle. Supporters of the valence bond and crystal field theories may not be so enthusiastic, for Dr. Rundle starts with the Lewis theory of valence-the inert gas rule and the electron pair bond-and describes how these concepts are modified by molecular orbital theory to explain electron deficient compounds, transition metal complexes and outer ' $d$ ' orbital compounds. He produces convincing arguments to show that the lastnamed are mis-named, and that such compounds as $\mathbf{S F}_{6}$, $\mathrm{PCl}_{5}$, and the polyhalides can all be explained by molecular orbital theory without using outer ' $d$ ' orbitals at all; the similarity of the explanations for $\mathrm{SF}_{6}$ and for the others might have been made clearer by giving the full molecular orbital diagram for $\mathrm{I}_{3}{ }^{-}$.
The fourth chapter on the "Metallocenes" by William F. Little is about twice as long as most of the others and contains three times as many references; it is sound. conscientious, and detailed.

Kenneth B. Wiberg writes on "Oxidation-Reduction Mechanisms in Organic Chemistry". After explaining the kinetic hydrogen isotope effect, he deals with the different types of mechanism, for example, hydride. hydrogen atom, and electron transfer. The explanations are clear and illustrated with many examples; perhaps more attention could have been paid to the differences between one and two electron oxidants.

"The Chemistry of Biological Energy Transfer" is at large subject. William P. Jencks starts well by explaining "the provincial dialect" used by biochemists and the role" of the high-energy bond. He describes the sources of useful energy in biological systems, and the extent of our rather scanty knowledge on how chemical energy is used in muscle contraction, the transport of electrolytes. the generation of electricity for nerve conduction, and bioluminescence. But his main section is on group transfer reactions; these are reactions whereby groups like phosphate or acyl are transferred from one compound to another, sometimes retaining an energy-rich bond arcl sometimes not. This section is like a high-quality valise' used as a cabin trunk. The excellent articles which appear from time to time in the Scientific American show that one needs plenty of space to explain biochemistry to the non-specialist. It is perhaps significant that while other contributors end their articles with summaries or conclusions the heading chosen by Dr. Jencks is "Future Developments".

The final article by Rudolf M. Salinger is on the Grignard reagent. One of the unresolved problems which used to puzzle the sophomore ten years ago was the structure of the Grignard reagent. Its nature, in ether at least, now appears to be clear, and the evidence is lucidly presented by Dr. Salinger. He also describes what is known about the mechanisms of its reactions. These will provide matcrial to puzzle sophomores and others for some time: to come.

Libraries have little choice; they will have to purchase this book. But, judging by the first volume, there can be little doubt that individuals will also wish to pay their sixty-four shillings. For the future, let us hope that the editor does not forget his preface. W. J. AlbERY

\section{ORGANOBORON CHEMISTRY}

\section{Organoboron Chemistry}

By Howard Steinberg. Vol. 1 : Boron-Oxygen and BoronSulfur Compounds. Pp. xxxii +950 . (New York and London: Interscience Publishers, a Division of John Wiley and Sons, Inc., 1964.) 248s.

\footnotetext{
TTHE author's title refers to all boron compounds containing carbon which is associated with other atoms, as in organic chemistry. Not only may the boron atom be attached to oxygen, which in turn is attached to a hydrocarbon group, but it may also be attached to the nitrogen atom of an amino-group; and, of course, the hydrocarbon group itself may carry a variety of atoms or groups. As boron is normally trivalent, and by coordination can be quadrivalent, and as three or four different groups can be attached to boron, the patterns must be as varied and subject to the same principles of order as the whole of organic chemistry. Not only are publications on organic compounds containing boron incroasing at a fantastic rate, but also the types of organic com. pounds becoming involved show that the whole of organic chemistry is coming under review with regard to the influence boron might have on the properties. To illust rate this trend, one needs only to mention as very recent
} 